\title{
ASSOCIATION OF POSTPRANDIAL BLOOD SUGAR WITH HYPERCOAGULABILITY IN COMPARISON TO FASTING BLOOD SUGARS IN DIABETIC AND HEALTHY PATIENTS: A CROSS-SECTIONAL STUDY
}

\author{
SRI RAMULU KADIYALA ${ }^{1}$, KARTHIK RAO ${ }^{2}$, RAO NR ${ }^{3}$, RAM BHAT ${ }^{2}$, JAYAPRAKASH RAO ${ }^{2}$, NAVIN ${ }^{4 *}$, BALAJI ${ }^{4}$ \\ ${ }^{1}$ Department of Cardiology, Kasturba Medical College, Manipal University, Manipal, Karnataka, India. ${ }^{2}$ Department of Internal Medicine, \\ Kasturba Medical College, Manipal University, Manipal, Karnataka, India. ${ }^{3}$ Consultant, Anugraha Medical Centre, Udupi, Karnataka, India. \\ ${ }^{4}$ Department of Pharmacology, Kasturba Medical College, Manipal University, Manipal, Karnataka, India. \\ Email: navin903@gmail.com
}

Received: 30 March 2017, Revised: 08 April 2017 Accepted: 13 April 2017

\section{ABSTRACT}

Objective: The aim of this study was to find the association of postprandial blood glucose with hypercoagulability in comparison to fasting blood sugars(FBS) in diabetic and healthy patients.

Methods: The present study involved a total of 156 patients, of which 78 were taken as cases (diabetics) and other 78 as controls (non-diabetics). Laboratory analysis included prothrombin time (PT), activated partial thromboplastin time (APTT), fibrinogen assay done along with fasting, and postprandial sugars.

Results: Platelets in diabetics and healthy controls were in normal range. Decrease in PT and partial thromboplastin time was noted in diabetics compared to non-diabetic controls. Fibrinogen levels were increased in cases compared to controls. Changes in PT values were more significant with postprandial blood sugar (PPBS) levels when compared to FBS levels, and APTT follows the same pattern with more in PPBS levels and FBS levels in diabetics. PPBS showed elevated fibrinogen when compared to FBS in diabetics as well as non-diabetics.

Conclusion: Type 2 diabetes mellitus is a hypercoagulable state as proven by the following results of our study.

Keywords: Hypercoagulation, Fibrinogen levels, Clotting factors, Diabetes mellitus.

(c) 2017 The Authors. Published by Innovare Academic Sciences Pvt Ltd. This is an open access article under the CC BY license (http://creativecommons. org/licenses/by/4. 0/) DOI: http://dx.doi.org/10.22159/ajpcr.2017.v10i7.18806

\section{INTRODUCTION}

Diabetes is chronic disease which is reaching an epidemic proportion in many parts of the world. Diabetes mellitus (DM) is a metabolic disorder characterized by elevated blood glucose levels and disturbances in carbohydrates, fats, and protein metabolism. Type 2 DM (T2DM) accounts for more than $90 \%$ of the diabetic population worldwide [1,2]. Both genetic and environmental factors are important in the development of the disease. The International Diabetes Federation estimates showed that 194 million people had diabetes in 2003 and it is expected to reach 333 million by the year of 2025 [1].

30 years ago, the prevalence of diabetes in India based on the Indian Council of Medical Research multicentric survey was around 2\% in urban India and $1 \%$ in rural India. In just three decades, these prevalence rates have shot up to $12-16 \%$ in urban India and 3-8\% in rural India, in adults over 20 years of age. These represent a $600-800 \%$ increase in prevalence rates of diabetes something which is unparalleled to any Western nation. Indeed, India is now referred to as the "diabetic capital" of the world.

Patients with diabetes are notorious for their risk of vascular events. Apart from the effects of diabetes and its prerequisite hyperglycemia on the development of atherosclerosis, this high risk may also be caused by the procoagulant state found in diabetes [3,4]. Type 2 diabetes is associated with a markedly increased risk for atherosclerotic coronary arteries and cerebrovascular diseases. Cardiovascular disease (CVD) remains the main cause of morbidity and mortality in individuals with diabetes. Up to $80 \%$ of diabetes, patients die because of cardiovascular complications, and the risk of atherothrombotic events in this population is similar to non-diabetic individuals with a history of ischemic heart disease (IHD) [3,5]. Moreover, the prognosis in these individuals following an event remains poor, despite major advances in treatment. Increased atherothrombotic risk is even evident in the prediabetes stage as patients with insulin resistance and normoglycemia are at risk of cardiovascular events secondary to clustering of risk factors [4].

The underlying mechanisms for increased thrombosis risk in diabetes are complex and involve multiple pathways. Patients with diabetes have premature atherosclerosis and more extensive vascular disease, predisposing them to plaque rupture and thrombus formation [6]. In addition, these individuals have increased thrombotic tendency due to platelet hyperreactivity and increased activation of prothrombotic coagulation factors coupled with decreased fibrinolysis [7].

In recent years, hyperglycemia per se, even without overt diabetes, has gained interest as a potential target to improve clinical outcomes in hospitalized patients with acute illness [8]. In addition, there is evidence that abnormalities during the postprandial state, specifically postprandial hyperglycemia, are independent risk factors for atherosclerosis. Recent epidemiological studies suggest postprandial hyperglycemia is an independent risk factor for CVD that has effects greater than that of fasting hyperglycemia $[9,10]$. In this setting, the effects of hyperglycemia on the coagulation system may be of greater importance than previously considered. Hence, the aim of this study was to find the association of postprandial blood glucose with hypercoagulability in comparison to fasting blood sugars (FBS) in diabetic and healthy patients. Hence, the aim is to study the coagulation parameters in patients with T2DM, to compare 
the obtained results with the control group (non-diabetic healthy controls), to study the relationship of coagulation parameters with fasting plasma glucose, and also to find the association of postprandial blood sugar with fibrinogen in comparison to FBS in diabetics.

\section{METHODS}

Outpatients attending Kasturba Hospital, Manipal, during the study period from November 2012 to August 2014, were screened and those who fulfilled the below mentioned inclusion criteria were selected. Others were excluded. This was a cross-sectional study. Cases were selected from outpatients attending Kasturba Hospital, Manipal, who were recently detected with DM based on the American Diabetes Association criteria. Controls were selected from our patients attending Kasturba Hospital, Manipal, with no history of DM or hypertension or IHD who were age and sex matched to the cases. The present study involved a total of 156 patients, of which 78 were taken as cases (diabetics) and other 78 as controls (non-diabetics) according to inclusion and exclusion criteria. Those patients who gave written consent for the study and fulfilled the inclusion and exclusion criteria were included in this study.

\section{Inclusion criteria}

Patients $>30$ years of age diagnosed to have T2DM. Patients with T2DM are not on treatment. Healthy controls who are matching diabetic patients

\section{Exclusion criteria}

The exclusion criteria were as follows: Type 1 diabetes and steroidinduced DM. Patients with known hypercoagulable disorders. Patients on anticoagulants. Patients in renal failure, liver disease and multiple organ dysfunction syndrome, and sepsis. History of alcohol consumption and smoking.

\section{Investigations}

FBS was sample collected in sugar vacutainer, after overnight fasting of $8 \mathrm{hrs}$, samples area analyzed by hexokinase method. Postprandial blood sugar (PPBS) sample was collected in sugar vacutainer after $2 \mathrm{hrs}$ of taking meal and done by hexokinase method. Prothrombin time (PT) sample was collected in sugar citrated tubes and done by mechanical method. Activated partial thromboplastin time (APTT) sample was collected in sugar citrated tubes and done by mechanical method. Fibrinogen was collected in sugar sodium citrate buffer and done by density analyzer. Glycated hemoglobin sample was collected in sugar ethylenediaminetetraacetic acid tubes and done by chromatography. PT, APTT, and fibrinogen done along with fasting and postprandial sugars on the same day.

\section{Statistical analysis}

Data were obtained and analyzed using SPSS 16 version software. $\mathrm{p}<0.05$ was taken as significant and was measured using Student paired t-test for the FBS and PPBS and PT, APTT, and fibrinogen. Analysis for adjusted sugar levels was done using Wilcoxon signed-rank test after adjusting for blood glucose levels by maintaining at least $40 \mathrm{mg} / \mathrm{dl}$ difference between FBS and PPBS in both diabetics and controls. Pearson's correlation coefficient was used to find the correlation between fibrinogen and FBS, PPBS.

\section{RESULTS}

\section{Sex distribution}

In diabetic patients, of the total 78 , a number of male patients were $63(81 \%)$ and female patients were 15 (19\%). In non-diabetic patients, of the total 78, a number of male patients were $62(79 \%)$ and female patients were 15 (21\%) (Fig. 1).

\section{Age and body mass index (BMI)}

The mean age of the patients in diabetic group was $52.474 \pm 10.327$ years and in non-diabetic group was $52.436 \pm 8.960$ years. The mean BMI in diabetic group was $24.561 \pm 2.652$ and in non-diabetic group was $23.389 \pm 2.406$ (Table 1$)$

\section{Blood pressure}

The mean systolic blood pressure in diabetic group was $129.026 \pm 11.499 \mathrm{mmHg}$ and in non-diabetic group was $128.692 \pm 16.356 \mathrm{mmHg}$. The mean diastolic blood pressure in diabetic group was $81.897 \pm 8.128 \mathrm{mmHg}$ and in non-diabetic group was $83.769 \pm 6.477$ mmHg (Fig. 2).

\section{Blood counts in diabetics and non-diabetics}

The mean of hemoglobin in diabetic group was $14.007 \pm 1.299$ and in non-diabetic group was $13.945 \pm 0.996$ (Fig. 3). The mean platelet count in diabetic group was $259576.9 \pm 62238.444$ and in non-diabetic group was $290192.3 \pm 54976.041$ (Fig. 4). The mean total leukocyte count in diabetic group was $7037.179 \pm 1787$ and in non-diabetic group was $7235.128 \pm 1652.874$ (Fig. 5).

\section{Liver function tests}

In both diabetic and non-diabetic group liver enzymes, aspartate transaminase, alanine transaminase, and alkaline phosphatase were within normal limits (Table 2).

\section{Serum creatinine level}

The mean serum creatinine level in diabetic group was $0.888 \pm 0.194$ and in non-diabetic group was $0.772 \pm 0.152$ (Fig. 6).

\section{Lipid profile}

In diabetic group, total cholesterol, triglyceride, and low-density lipoprotein levels were elevated when compared to non-diabetic group. There was significant difference in triglyceride level in diabetic and non-diabetic group with $\mathrm{p}<0.01$. The high-density lipoprotein levels were elevated in non-diabetic group when compared to the diabetic group $\mathrm{p}<0.001$ (Table 3 and Fig. 7).

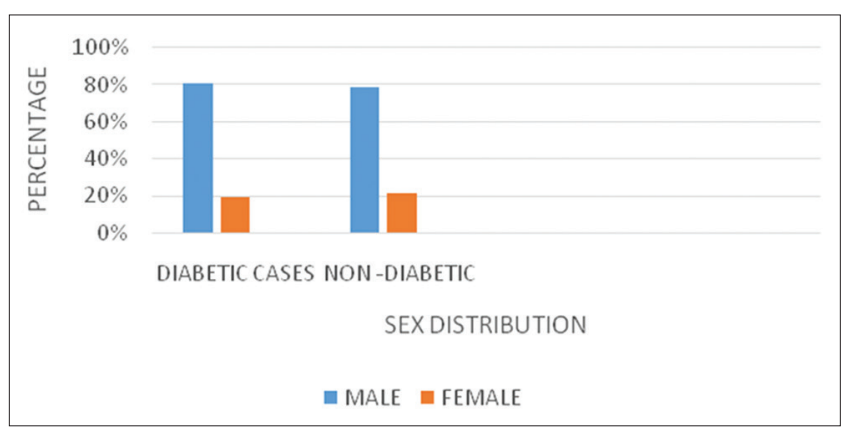

Fig. 1: Sex distribution in diabetics and non-diabetics

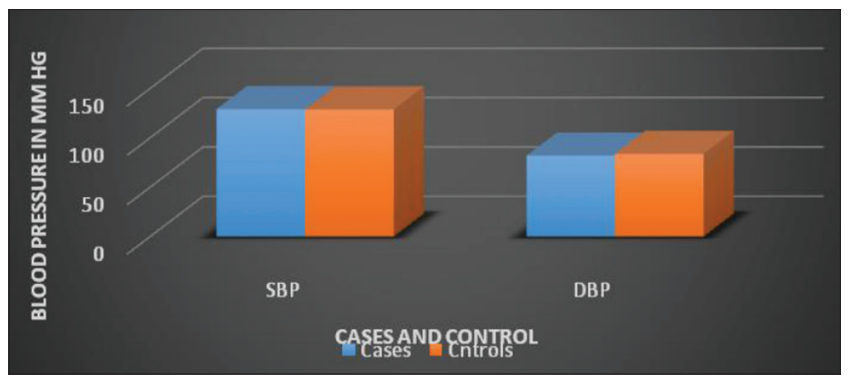

Fig. 2: Mean blood pressure in cases and controls

Table 1: Age and body mass index in diabetics and non-diabetics

\begin{tabular}{lll}
\hline Mean & Diabetics & Non-diabetics \\
\hline Age of patients & $52.474 \pm 10.327$ & $52.436 \pm 8.960$ \\
Body mass index & $24.561 \pm 2.652$ & $23.389 \pm 2.406$ \\
\hline
\end{tabular}




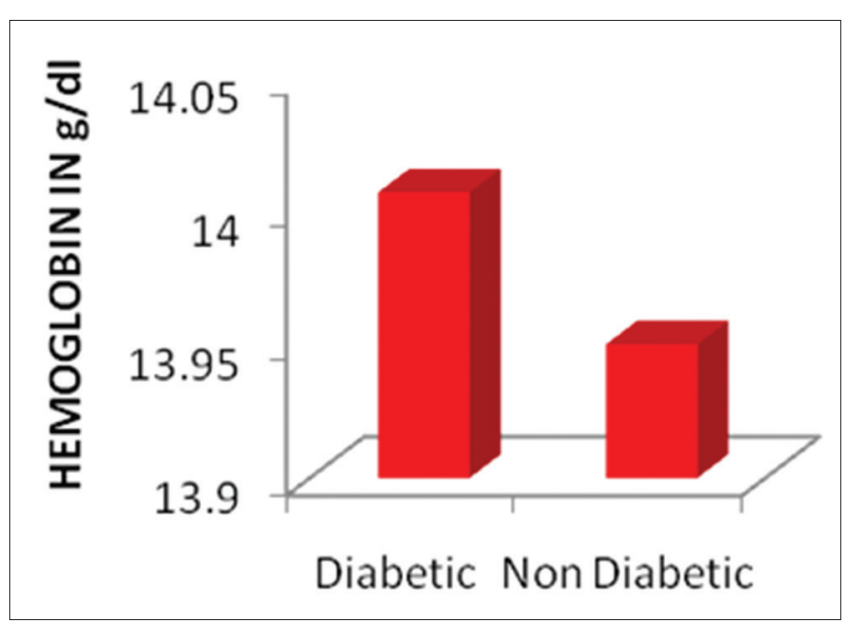

Fig. 3: Mean hemoglobin values

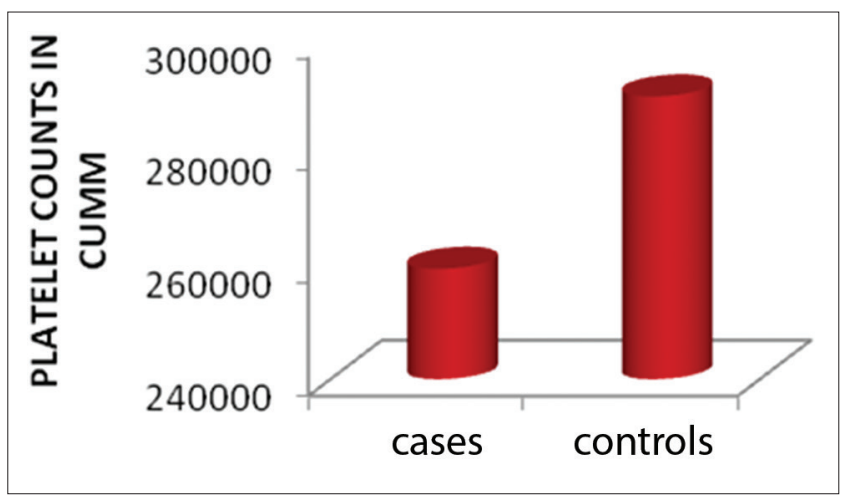

Fig. 4: Mean platelet counts

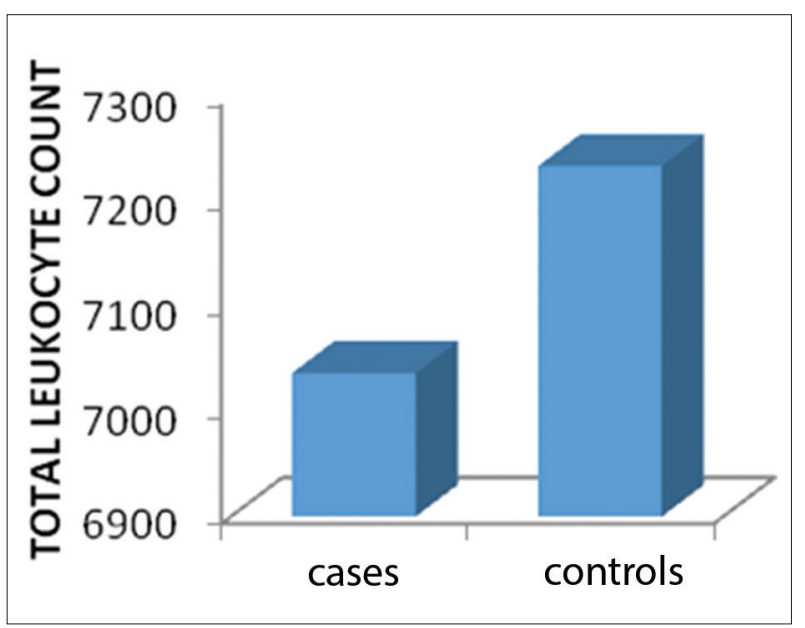

Fig. 5: Mean total leukocyte count

Glycated hemoglobin

The mean glycated hemoglobin level in diabetic group was $9.274 \pm 2.28$ and in non-diabetic group was $5.550 \pm 0.445$ with $\mathrm{p}=0.001$ (Fig. 8).

\section{Blood sugar}

In diabetic group, the mean of FBS was $184.09 \pm 64.52$ and the mean of postprandial blood sugar was $265.52 \pm 78.00$ (Fig. 9). In the non-diabetic group, the mean of FBS was $89.62 \pm 8.9$ and mean of postprandial blood sugar was $121.41 \pm 10.18$ (Fig. 10).

\section{Fasting PT/APTT}

The mean fasting PT in diabetic group was $15.01 \pm 1.95$ seconds and in non- diabetic group was $15.30 \pm 1.23$ seconds with ( $\mathrm{p}=0.04$ ) (Fig. 11).

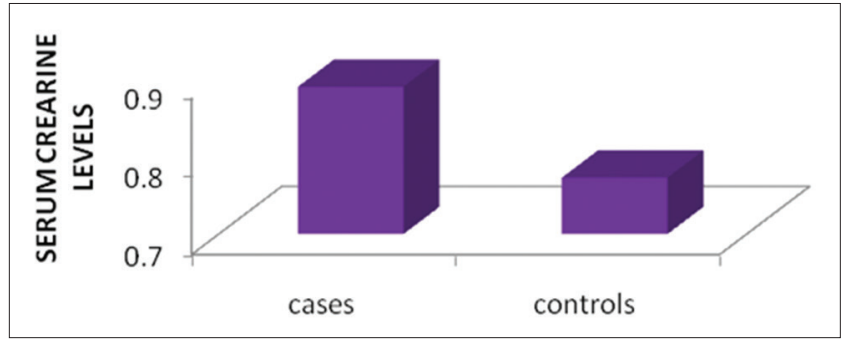

Fig. 6: Mean serum creatinine level

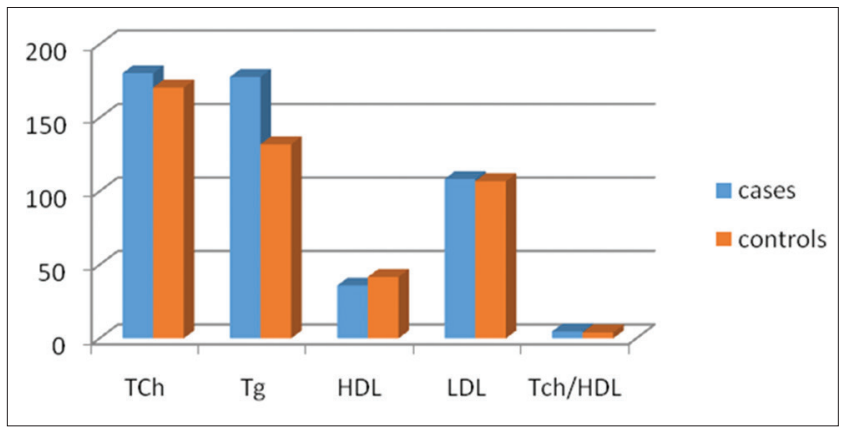

Fig. 7: Lipid profile analysis

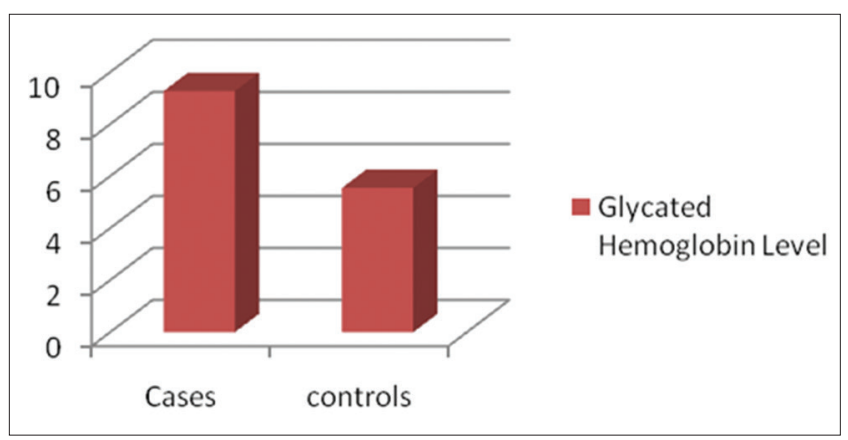

Fig. 8: Glycated hemoglobin level

Table 2: Liver function test in diabetics and non-diabetics

\begin{tabular}{lll}
\hline Mean & Diabetics & Non-diabetics \\
\hline AST & $26.179 \pm 8.460$ & $25.333 \pm 6.418$ \\
ALT & $29.603 \pm 11.906$ & $25.590 \pm 7.968$ \\
ALP & $93.244 \pm 21.826$ & $97.218 \pm 20.836$ \\
\hline \multicolumn{2}{l}{ AST: Aspartate transaminase, ALT: Alanine transaminase, ALP: Alkaline } \\
phosphatase
\end{tabular}

Table 3: Lipid profile in diabetics/non-diabetics

\begin{tabular}{lll}
\hline Mean & Diabetics & Non-diabetics \\
\hline Cholesterol & $180.35 \pm 36.06$ & $167.60 \pm 33.19$ \\
Triglyceride & $177.79 \pm 108.22$ & $132.01 \pm 55.79$ \\
HDL & $35.94 \pm 11.81$ & $41.84 \pm 14.64$ \\
LDL & $108.38 \pm 28.26$ & $106.91 \pm 27.92$ \\
Ratio of total cholesterol/HDL & $4.67 \pm 1.49$ & $4.07 \pm 1.46$ \\
\hline \multicolumn{2}{l}{ HDL: High-density lipoprotein, LDL: Low-density lipoprotein }
\end{tabular}

The mean fasting APTT in diabetic group was $30.57 \pm 4.17$ seconds and in non-diabetic group was $32.10 \pm 4.16$ seconds with $(\mathrm{p}=0.02)$ (Fig. 12).

\section{Fasting fibrinogen assay}

The mean fasting fibrinogen level in diabetic group was $356.37 \pm 126.95$ and in non-diabetic group was $282.95 \pm 63.47$ $(\mathrm{p}=0.01)$ (Fig. 13). 


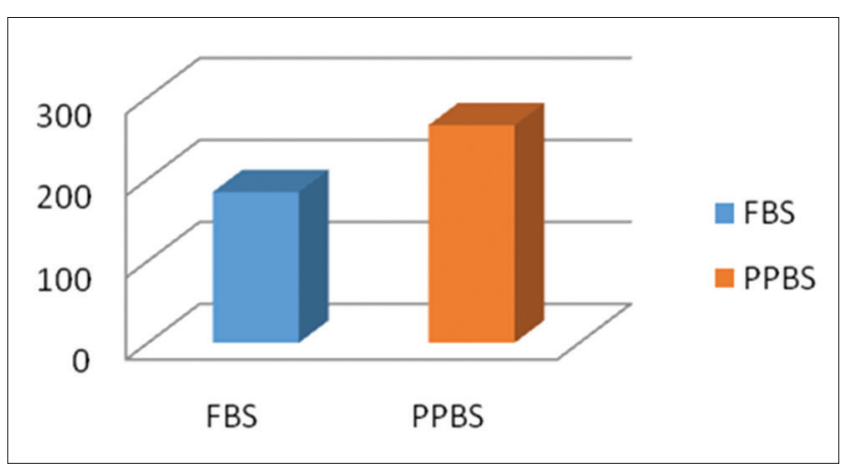

Fig. 9: Fasting and postprandial blood sugar in diabetics

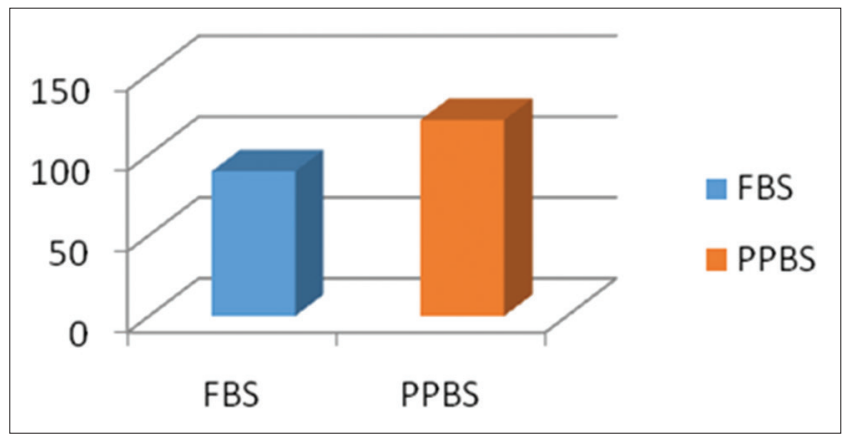

Fig. 10: Fasting and postprandial blood glucose in non-diabetics

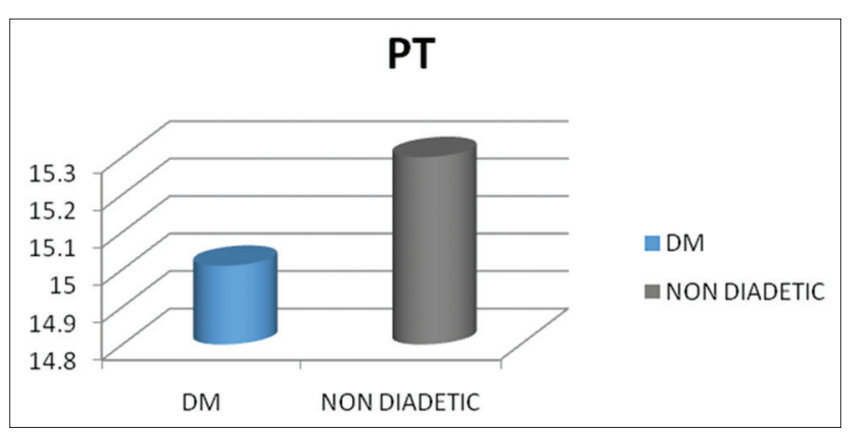

Fig. 11: Prothrombin time in diabetics versus non-diabetics

PT in fasting and postprandial blood sugars in diabetics/nondiabetics

In the diabetic group, the mean fasting PT was $15.01 \pm 1.95$ seconds and the mean postprandial prothrombin time was $13.95 \pm 1.01$ seconds with $\mathrm{p}=0.04$. In non-diabetic group, the mean fasting PT was $15.30 \pm 1.23$ seconds and the mean postprandial PT was $15.10 \pm 1.07$ seconds with p=0.686 (Figs. 14 and 15).

APTT in fasting and postprandial blood sugars in diabetics/nondiabetics

In the diabetic group, the mean fasting activated thromboplastin time was $30.57 \pm 4.17$ seconds and mean postprandial activated thromboplastin time was $29.32 \pm 5.10$ seconds with $\mathrm{p}=0.02$. In nondiabetic group, the mean fasting activated thromboplastin time was $32.10 \pm 4.16$ seconds and mean postprandial activated thromboplastin time was $30.91 \pm 4.04$ seconds with $\mathrm{p}=0.1$ (Figs. 16 and 17).

Fibrinogen in fasting and postprandial blood sugars in diabetics/ non-diabetics

In diabetic group, the mean fasting fibrinogen assay was $356.37 \pm 126.95$ and the mean postprandial fibrinogen assay was $374.45 \pm 130.49$ with $\mathrm{p}=0.001$. In non-diabetic group, the mean fasting fibrinogen assay was $282.95 \pm 63.47$ and mean postprandial fibrinogen assay was

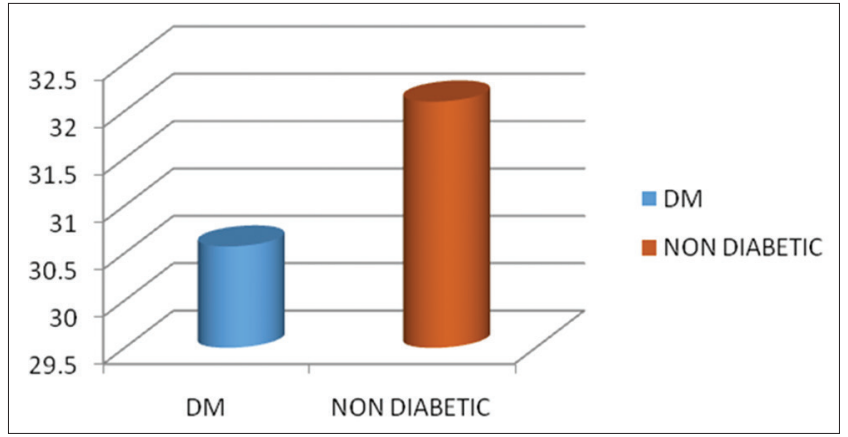

Fig. 12: Activated partial thromboplastin time in diabetics versus non-diabetics

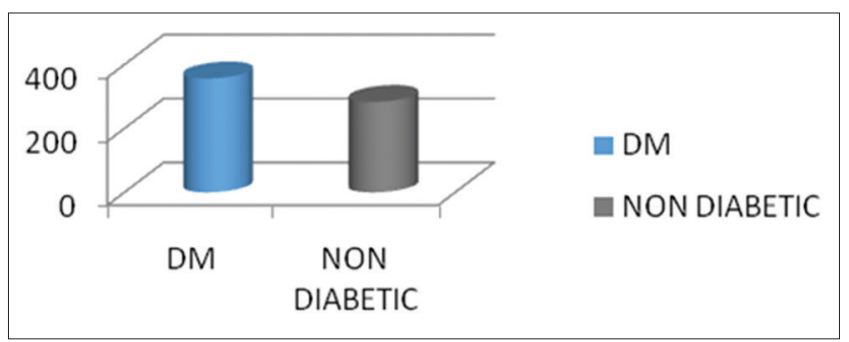

Fig. 13: Free fibrinogen assay in diabetics versus non-diabetics

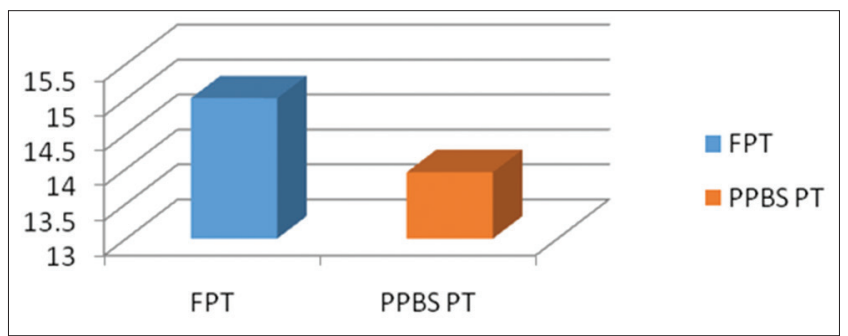

Fig. 14: Fasting and postprandial prothrombin time in diabetic patients

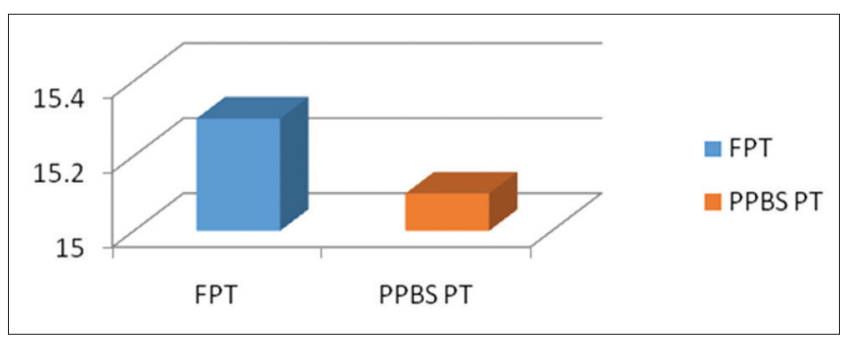

Fig. 15: Fasting and postprandial prothrombin time in non-diabetics

$306.95 \pm 66.67$ with $\mathrm{p}=0.002$. Pearson's correlation is given in table. In diabetics, fibrinogen correlation with PPBS is $r=0.3$, when compared to FBS $r=0.2$, and in non-diabetics, fibrinogen correlation with PPBS is $r=0.1$, when compared to FBS $r=0.08$ (Figs. 18-20 and Table 4).

Significance was found in adjusted blood sugars for fibrinogen between fasting and PPBS in both diabetics and non-diabetics (Table 5).

\section{DISCUSSION}

DM is associated with an increased risk of atherosclerosis, hence considered as a procoagulant state. Few studies are available evaluating coagulation screening tests in diabetes. In the present study, we aim to investigate coagulation parameter changes in diabetes and the 


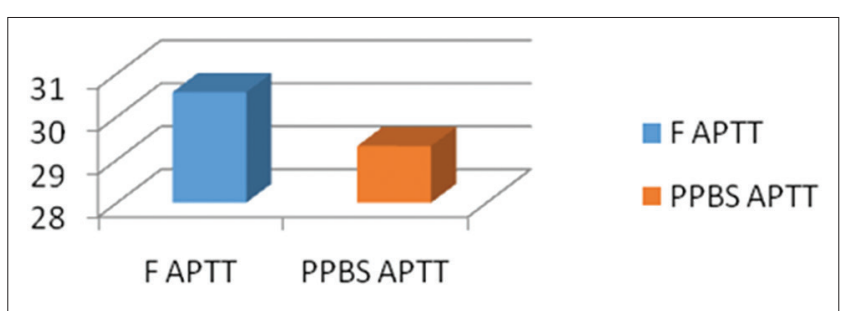

Fig. 16: Fasting and postprandial activated partial thromboplastin time in diabetics

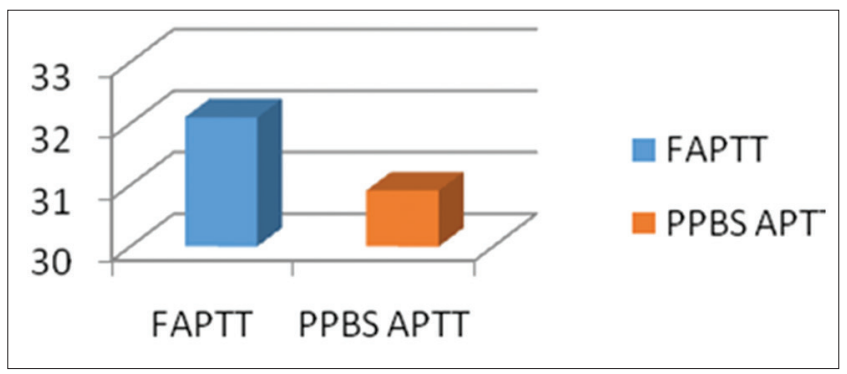

Fig. 17: Fasting and postprandial activated partial thromboplastin time in non-diabetics

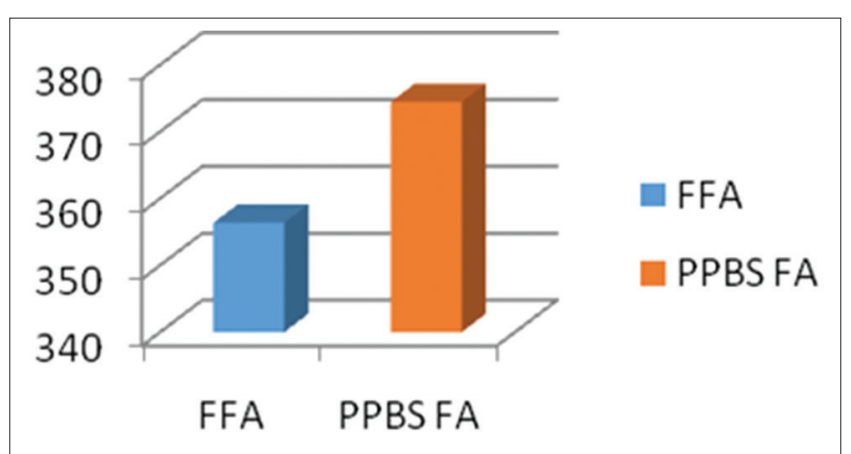

Fig. 18: Fasting and postprandial free fibrinogen assay in diabetics

association of PPBS with hypercoagulability as compared to FBS. The diabetic patients and healthy controls were age and sex matched. In the present study, the mean age of the diabetics was $52.47 \pm 10.32$ years and healthy controls was $52.43 \pm 8.9$ years. The male-to-female ratio was $4: 1$ in both the diabetic and non-diabetic groups.

The mean BMI in diabetes was $24.56 \pm 2.65$ and in healthy controls was $23.38 \pm 2.4$. The platelet count was normal in the diabetic patients, i.e., there is no quantitative change compared to the non-diabetic population [3]. Platelet counts have been found to be normal in studies done by Borsey et al. [11] and Erem et al. [12]. In the study done by Madan et al., [13] diabetics have normal platelets counts similar to the present study where platelets in diabetics and healthy controls were in normal range (Table 6). Hyperglycemia directly contributes to endothelial injury through irreversible glycation of collagen and other subendothelial structural proteins of the vessel, forming advanced glycation end products [14] and endothelial dysfunction, can lead to an activated state characterized, in part, by increased platelet adhesion and aggregations [15].

Very few reports are available regarding coagulation screening tests in diabetes. The previous studies which were done for coagulation tests in diabetics and healthy controls were done in fasting state. Metabolic syndrome and T2DM patients have increased Factor VII (FVII) levels which can lead to decrease in PT [3,4]. Increased levels of TF in T2DM patients activate Factor VII and changed into VIIa. Activated Factor VII

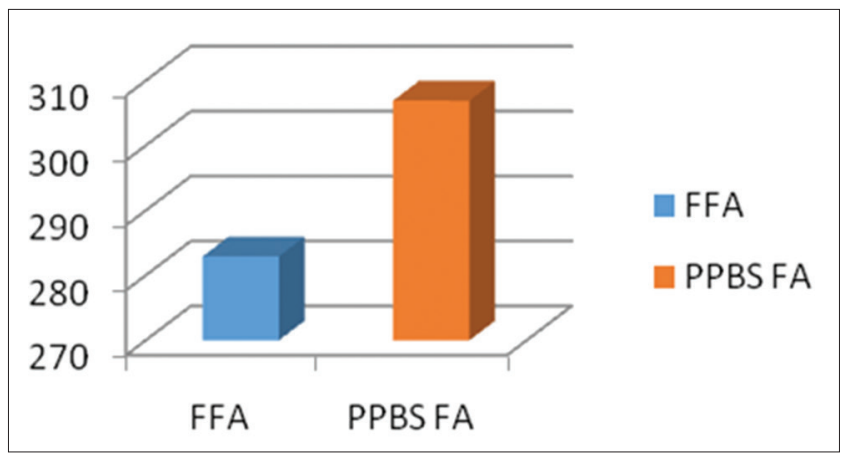

Fig. 19: Fasting and postprandial free fibrinogen assay in non-diabetics

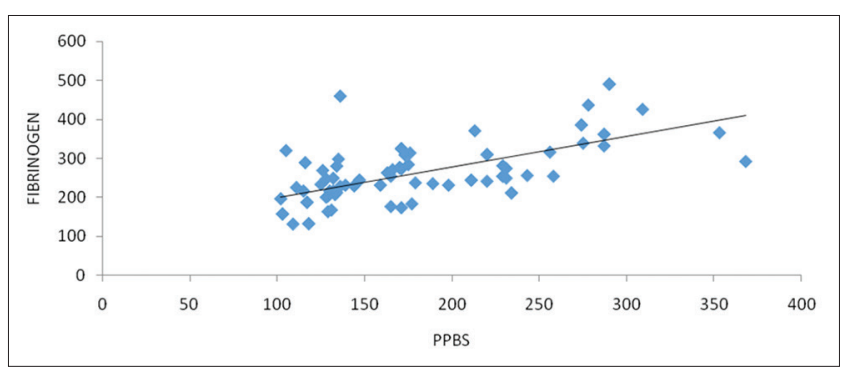

Fig. 20: Pearson's correlation for fibrinogen levels and postprandial blood sugar in diabetics versus non-diabetics

Table 4: Pearson's correlation for fibrinogen levels in diabetics versus non-diabetics

\begin{tabular}{lll}
\hline Correlation & Diabetic fibrinogen & Non-diabetic fibrinogen \\
\hline FBS & $\mathrm{r}=0.2$ & $\mathrm{r}=0.08$ \\
PPBS & $\mathrm{r}=0.3$ & $\mathrm{r}=0.1$ \\
\hline
\end{tabular}

PPBS: Postprandial blood sugar, FBS: Fasting blood sugars

Table 5: Adjusted blood sugar levels

\begin{tabular}{lll}
\hline Mean & DM & Healthy controls \\
\hline Fasting fibrinogen & $371.15 \pm 156.21$ & $285.3 \pm 44.66$ \\
Postprandial fibrinogen & $396.8 \pm 155.96$ & $305.3 \pm 44.56$ \\
$\mathrm{p}$ & 0.001 & 0.001 \\
\hline
\end{tabular}

DM: Diabetes mellitus

Table 6: Comparison of platelet counts between the present study and previous studies

\begin{tabular}{lll}
\hline Mean & Diabetics (platelet counts) & Non-diabetics \\
\hline Madan et al. & $2.02 \pm 0.61$ & $2.44 \pm 0.63$ \\
Present study & $2.59 \pm 0.62$ & $2.90 \pm 0.54$ \\
\hline
\end{tabular}

(VIIa) triggers the extrinsic pathway of coagulation through converting to Xa. In the study done by Acang and Jalil [16] showed decreased PT in diabetics when compared to healthy controls, similar results shown in the present study $(\mathrm{p}<0.05)$ (Table 7). The Factor VIII/vWF complex is also increased in individuals with insulin resistance and T2DM $(4,52$, $59,61)$, which leads to decrease in APTT. The study done by Zhao et al. [17] showed significant difference in APTT in diabetics and controls being decreased APTT in diabetics when compared to healthy controls, similar results were obtained in the present study $(\mathrm{p}=0.02)$ (Table 8). Fibrinogen, another acute phase reactant, is increased in diabetics $[4,18,19]$. An increase in plasma fibrinogen levels is also considered an independent risk factor for CVD [20]. The effect of hyperglycemia on the 
hemostatic system is observed in the fibrinogen molecule. The glycation of fibrinogen results in the formation of a denser fibrin clot with finer fibers that is resistant to fibrinolysis. The glycated fibrin binds less to both tissue-type plasminogen activator and plasminogen and generates less plasmin but binds more to alpha2-antiplasmin [3,4]. In the study done by Madan et al. [13] and by Acang and Jalil [16] showed significant difference in fibrinogen levels between diabetic and healthy patients with increase in fibrinogen in diabetics, similar to the result obtained in the present study $(\mathrm{p}=0.03)$ (Table 9).

An acute increase in clotting Factor VII has been described during induced hyperglycemia in both diabetic and healthy patients [21] while an enhancement of thrombin activity has been shown in the postprandial phase in Type 2 diabetic patients; this was proportional to the level of hyperglycemia [22]. Postprandial hypertriglyceridemia has been found to precede the activation of coagulation Factor VII, and the degree of Factor VII activation is proportional to the increase in plasma triglycerides [23]. Since there is extensive evidence of important interactions between plasma lipoproteins and coagulation, including platelet aggregation and fibrinolysis [24], it seems reasonable that there could be an increased thrombotic tendency in the postprandial phase.

In the present study, changes in PT values was more significant with PPBS levels $(\mathrm{p}=0.04)$ when compared to FBS levels and APTT follows the same pattern with more in PPBS levels $(\mathrm{p}=0.02)$ and FBS levels. In the present study, in non-diabetes, changes in PT values were not significant with PPBS levels when compared to FBS levels $(p=0.6)$ and APTT follows the same pattern with more in PPBS levels and FBS levels $(\mathrm{p}=0.1)$.

At present, to the best of our knowledge, there are no studies available in literature to compare the coagulation parameters PT, APTT in fasting, and postprandial state. The study done in diabetic patients by Temelkova-Kurktschiev et al. [25] showed significant elevation of fibrinogen levels in the postprandial state. In their study, the postprandial phase fibrinogen and PPBS were significantly associated $(\mathrm{p}<0.05)$ and the association with FBS and fibrinogen was found to be not significant, which was similar to the result obtained in the present study with PPBS showed elevated fibrinogen $(\mathrm{p}=0.001)$ when compared to FBS. The synthesis of fibrinogen, a strong risk factor for CVD in both diabetic and non-diabetic patients [26], increases in the postprandial

Table 7: Comparison of prothrombin time between the present study and previous studies

\begin{tabular}{lll}
\hline Mean & DM & Healthy controls \\
\hline Acang and Jalil & $10.1 \pm 1.31$ & $11.04 \pm 0.93$ \\
Present study & $15.01 \pm 1.95$ & $15.3 \pm 1.23$ \\
\hline
\end{tabular}

DM: Diabetes mellitus

Table 8: Comparison of APTT between the present study and previous studies

\begin{tabular}{lll}
\hline Studies & DM & Healthy controls \\
\hline Zhao & $26.9 \pm 6.2$ & $27.8 \pm 6.2$ \\
Present study & $30.57 \pm 4.17$ & $32.10 \pm 4.16$ \\
\hline
\end{tabular}

DM: Diabetes mellitus, APTT: Activated partial thromboplastin time

Table 9: Comparison of fibrinogen between the present study and previous studies

\begin{tabular}{lll}
\hline Studies & DM & Healthy controls \\
\hline Madan & $252 \pm 40.23$ & $227.5 \pm 22.8$ \\
Acang and Jalil & $442.42 \pm 86.79$ & $349.2 \pm 35.26$ \\
Present study & $356.37 \pm 126.95$ & $282.95 \pm 63.47$ \\
\hline
\end{tabular}

DM: Diabetes mellitus state in diabetic patients. In controls, also, the present study showed elevated fibrinogen in the postprandial state $(\mathrm{p}=0.001)$ when compared to fasting state.

\section{Limitations}

Limitations Postprandial lipid profile and anti-fibrinolysis analysis could not be done in view of cost constraints. Another limitation was small sample size.

\section{CONCLUSIONS}

T2DM is a hypercoagulable state as proven by the following results of our study which states PT was decreased in the diabetics when compared to controls, APTT was decreased in diabetics when compared to controls, fibrinogen levels were increased in diabetics when compared to controls. The postprandial blood sugar levels were significantly associated with elevated fibrinogen levels in both diabetics and non-diabetics when compared to the FBS levels indicating a more significance association of postprandial state with hypercoagulability.

\section{REFERENCES}

1. Tripathy B, Chandalia HB, Das AK, Rao P. RSSDI: Textbook of Diabetes Mellitus. New Delhi: JP Medical Ltd; 2012.

2. Jain R, Jain P, Jain P. A review on treatment and prevention of diabetes mellitus. Int J Curr Pharm Res 2016;8(3):16-8.

3. Carr ME. Diabetes mellitus: A hypercoagulable state. J Diabetes Complications 2001;15(1):44-54

4. Grant PJ. Diabetes mellitus as a prothrombotic condition. J Intern Med 2007;262(2):157-72.

5. Haffner SM, Lehto S, Ronnemaa T, Pyorala K, Laakso M. Mortality from coronary heart disease in subjects with Type 2 diabetes and in nondiabetic subjects with and without prior myocardial infarction. $\mathrm{N}$ Engl J Med 1998;339(4):229-34.

6. Stratmann B, Tschoepe D. Atherogenesis and atherothrombosis - Focus on diabetes mellitus. Best Pract Res Clin Endocrinol Metab 2009;23(3):291-303.

7. Grant PJ. Inflammatory, atherothrombotic aspects of Type 2 diabetes. Curr Med Res Opin 2005;21 Suppl 1:S5-12.

8. van den Berghe G, Wouters P, Weekers F, Verwaest C, Bruyninckx F, Schetz $\mathrm{M}$, et al. Intensive insulin therapy in critically ill patients. N Engl J Med 2001;345(19):1359-67.

9. Hanefeld M, Fischer S, Julius U, Schulze J, Schwanebeck U, Schmechel $\mathrm{H}$, et al. Risk factors for myocardial infarction and death in newly detected NIDDM: The Diabetes Intervention Study, 11-year follow-up. Diabetologia 1996;39(12):1577-83.

10. Kuusisto J, Mykkänen L, Pyörälä K, Laakso M. NIDDM and its metabolic control predict coronary heart disease in elderly subjects. Diabetes 1994;43(8):960-7.

11. Borsey DQ, Prowse CV, Gray RS, Dawes J, James K, Elton RA, et al. Platelet and coagulation factors in proliferative diabetic retinopathy. J Clin Pathol 1984;37(6):659-64

12. Erem C, Hacihasanoglu A, Celik S, Ovali E, Ersöz HO, Ukinç K, et al. Coagulation and fibrinolysis parameters in Type 2 diabetic patients with and without diabetic vascular complications. Med Princ Pract 2005;14(1):22-30.

13. Madan R, Gupt B, Saluja S, Kansra UC, Tripathi BK, Guliani BP. Coagulation profile in diabetes and its association with diabetic microvascular complications. J Assoc Physicians India 2010:58:481-4.

14. Tan KC, Chow WS, Ai VH, Metz C, Bucala R, Lam KS. Advanced glycation end products and endothelial dysfunction in Type 2 diabetes. Diabetes Care 2002;25(6):1055-9.

15. Keating FK, Sobel BE, Schneider DJ. Effects of increased concentrations of glucose on platelet reactivity in healthy subjects and in patients with and without diabetes mellitus. Am J Cardiol 2003;92(11):1362-5.

16. Acang N, Jalil FD. Hypercoagulation in diabetes mellitus. Southeast Asian J Trop Med Public Health 1993;24 Suppl 1:263-6.

17. Zhao Y, Zhang J, Zhang J, Wu J. Diabetes mellitus is associated with shortened activated partial thromboplastin time and increased fibrinogen values. PLoS One 2011;6(1):e16470.

18. Weiler H, Isermann BH. Thrombomodulin. J Thromb Haemost 2003;1(7):1515-24

19. Yudkin JS. Abnormalities of coagulation and fibrinolysis in insulin resistance. Evidence for a common antecedent? Diabetes Care 1999;22 Suppl 3:C25-30 
20. Juhan-Vague I, Alessi MC, Vague P. Thrombogenic and fibrinolytic factors and cardiovascular risk in non-insulin-dependent diabetes mellitus. Ann Med 1996;28(4):371-80.

21. Ceriello A, Giugliano D, Quatraro A, Dello Russo P, Torella R. Blood glucose may condition factor VII levels in diabetic and normal subjects. Diabetologia 1988;31(12):889-91.

22. Ceriello A, Taboga C, Tonutti L, Giacomello R, Stel L, Motz E, et al. Post-meal coagulation activation in diabetes mellitus: The effect of acarbose. Diabetologia 1996;39(4):469-73

23. Silveira A, Karpe F, Johnsson H, Bauer KA, Hamsten A. In vivo demonstration in humans that large postprandial triglyceriderich lipoproteins activate coagulation factor VII through the intrinsic coagulation pathway. Arterioscler Thromb Vasc Biol 1996;16(11):1333-9.

24. Gasser JA, Betteridge DJ. Lipids and thrombosis. Baillieres Clin Endocrinol Metab 1990;4(4):923-38.

25. Temelkova-Kurktschiev TS, Koehler C, Henkel E, Leonhardt W, Fuecker K, Hanefeld M. Postchallenge plasma glucose and glycemic spikes are more strongly associated with atherosclerosis than fasting glucose or HbA1c level. Diabetes Care 2000;23(12):1830-4.

26. Bruttomesso D, Iori E, Kiwanuka E, Zanetti M, Pianta A, Vettore M, et al. Insulin infusion normalizes fasting and post-prandial albumin and fibrinogen synthesis in Type 1 diabetes mellitus. Diabet Med 2001;18(11):915-20. 